\title{
Development of a Boundary Element Method-based numerical wave tank technique for the prediction of nonlinear wave kinematics and dynamics around offshore structures
}

\author{
H. G. Sung \\ Maritime and Ocean Engineering Research Institute, KORDI, Korea
}

\begin{abstract}
This paper presents recent accomplishments of the development of the Boundary Element Method (BEM) for nonlinear waves around offshore structures and resulting wave forces. In order to investigate the capability of the present method, the nonlinear diffraction problem of a truncated circular cylinder is simulated. It is found that the present method is fairly accurate for wave forces and run-ups when compared with experimental results and also with other numerical results.
\end{abstract}

Keywords: BEM, nonlinear waves, offshore structures, nonlinear diffraction.

\section{Introduction}

Nowadays, activities for marine gas and oil exploration move continuously into deeper ocean year by year. In order to design offshore structures for ultra deep sea, wave forces on offshore structures must be predicted with high accuracy. A number of theoretical, numerical and experimental studies have been developed for this purpose, but it seems that there still remains uncertainty to some extent.

In order to deal with nonlinear waves and resulting wave forces for high waves, it is necessary to apply a nonlinear method rather than the conventional linear theory. It is debatable whether nonlinear solutions based on the perturbation method are applicable for steep waves. It is also obvious that experimental studies in offshore basins are expensive for the preliminary design stage. Therefore, it is commonly accepted that numerical methods are appropriate for evaluating nonlinear wave forces on a body in large waves. Thus 
the present study is focused upon the numerical method. Hence the present paper is aimed to investigate the capability of the present numerical method, namely, the so-called a BEM-based numerical wave tank technique (Boo and Kim [1], Kim [10]), for the prediction of nonlinear wave forces and run-ups on offshore structures.

The numerical methods widely used in practice are based upon the usual assumption of an ideal fluid, and higher-order boundary elements as a field solver. This is partially justified because most successes in simulating nonlinear free surface waves have been relying upon boundary elements. It is also known as the numerical wave tank technique, which is conceived as a promising tool for predicting the nonlinear hydrodynamic behavior of offshore structures (Kim [10]).

Since the pioneering work of Longuet-Higgins and Cokelet [13] on spatially periodic nonlinear waves in two dimensions, many studies have been made on this kind of problems. To name a few, Dommermuth et al. [3] successfully calculated plunger-type waves within the framework of potential theory. Xü [22] investigated the kinematics of three-dimensional plunging breakers by using biquadratic boundary elements as an extension of Longuet-Higgins and Cokelet [13]. Nonlinear diffraction forces are reported in the literature such as Ferrant [4, 5], Boo and Kim [1], etc. Grilli and his colleagues have been trying to develop an efficient BEM for nonlinear wave evolution as shown in Grilli et al. [8], Guyenne and Grilli [9], and Grilli et al. [7], etc.

It is worthwhile to mention the paper written by Kristiansen et al. [12] in connection with the present paper. They tried to verify the conventional design tool of BEM in predicting diffracted wave elevation around a vertical circular cylinder. Applicability and shortcomings of the method were suggested.

The present paper is organized as follows. First, we present the mathematical formulation of the model with numerical formulation. Finally, we apply the method to the problem of nonlinear waves around the truncated circular cylinder. We compare the numerical results with those of experiments and other computation.

\section{Mathematical formulation}

We assume the fluid is incompressible and non-viscous, and also the flow is irrotational. So, we can define the velocity potential, as the scalar function, $\Phi(\vec{x}, t)$, of spatial variables, $\vec{x} \equiv(x, y, z)$, and time variable, $t$. The velocity potential is related to the fluid velocity vector, $\vec{u}=(u, v, w)$ by $\vec{u}=\nabla \Phi$, where $\nabla$ means the gradient operator. Thus, mass conservation becomes Laplace's equation for the potential in the fluid domain, $\Omega(t)$,

$$
\nabla^{2} \Phi(\vec{x}, t)=0
$$

The boundary of the fluid domain is composed of the free surface, body boundary, and far field boundaries. Appropriate boundary conditions (i.e., Dirichlet or Neumann) are stated in the below.

The kinematic and dynamic free surface conditions are expressed in an Eulerian representation as, 


$$
\begin{array}{r}
\frac{\partial \zeta}{\partial t}=-\nabla_{H} \Phi \cdot \nabla_{H} \zeta+\frac{\partial \Phi}{\partial z} \\
\frac{\partial \Phi}{\partial t}=-g z-\frac{1}{2}|\nabla \Phi|^{2}-\frac{p_{a}}{\rho}
\end{array}
$$

where $\zeta$ denotes the vertical position of the free surface as a function of the horizontal position and time, say, $\zeta=\zeta(x, y, t)$. The operator $\nabla_{H}$ means the horizontal component of the gradient operator. The constant $g$ is the gravitational acceleration and $\rho$ the fluid density. $p_{a}$ is the atmospheric or applied pressure on the free surface. Due to the local movement of the collocation points, boundary condition (3a) must be modified as follows.

$$
\frac{d}{d t} \Phi(\vec{x}, t)=\frac{\partial}{\partial t} \Phi(\vec{x}, t)+\frac{\partial \zeta}{\partial t} \frac{\partial \Phi}{\partial z}=-g z-\frac{1}{2}|\nabla \Phi|^{2}-\frac{p_{a}}{\rho}+\left[-\nabla_{H} \Phi \cdot \nabla_{H} \zeta+\frac{\partial \Phi}{\partial z}\right] \frac{\partial \Phi}{\partial z}
$$

The body boundary condition states that the normal velocity of the fluid particle on the body boundary is equal to the normal component of the velocity of the body,

$$
\nabla \Phi \cdot \vec{n}=\vec{V} \cdot \vec{n}
$$

where $\vec{n}=\left(n_{x}, n_{y}, n_{z}\right)$ is the outwards unit normal vector, and $\vec{V}$ is the body velocity, which is either given by the specified motion of the disturbance or obtained from the body equations of motion. This condition can also be used for wave-making boundary in the vertical upstream side. On the bottom boundary and other stationary parts of solid boundary, no-flow boundary condition is prescribed.

\section{Solution methodology}

\subsection{Boundary element method}

The bi-quadratic BEM of Sung [18] and Sung et al. [19] is utilized in numerical solution of the governing equation, Eq. (1), with time-dependent nonlinear free surface boundary conditions Eqs. (2) and (3). In this paper, we briefly restate the principal features of the method and its implementation.

In the first place, the Green's second identity transforms Laplace equation into the well-known boundary integral equation,

$$
\alpha(\vec{x}) \Phi(\vec{x})=\int_{\Gamma}\left[\Phi(\vec{\xi}) \frac{\partial G}{\partial n}(\vec{\xi}, \vec{x})-\frac{\partial \Phi}{\partial n}(\vec{\xi}) G(\vec{\xi}, \vec{x})\right] d \Gamma_{\xi}
$$

where $\alpha(\vec{x})$ is the normalized interior solid angle at point $\vec{x}, G(\vec{\xi}, \vec{x})=1 / 4 \pi r$ is the free-space Green's function, where $r=|\vec{r}|=|\vec{\xi}-\vec{x}|$ is the distance from the source point $\vec{\xi}$ to the field point $\vec{x}$. An analytic expression for the solid angle can be obtained by considering a trivial solution of the Laplace equation, to say a constant function, as $\alpha(\vec{x})=\int_{\Gamma} \partial G(\vec{\xi}, \vec{x}) / \partial n_{\xi} d \Gamma_{\xi}$. This expression is substituted into the boundary integral equation to obtain an improved form of the integral equation as follows. 


$$
\int_{\Gamma}\left\{[\Phi(\vec{\xi})-\Phi(\vec{x})] \frac{\partial G}{\partial n}(\vec{\xi}, \vec{x})-\frac{\partial \Phi}{\partial n}(\vec{\xi}) G(\vec{\xi}, \vec{x})\right\} d \Gamma_{\xi}=0
$$

It can be seen that the singularity of the first term is reduced by order of one, which means from the numerical point of view, all the integrations become regular through this improvement.

From the accuracy point of view, the higher the order of elements becomes, the more accurate the obtained solution will be. Practice, however, compels us to take a compromising order of approximation. As a result, "bi-quadratic elements" using $3 \times 3$ nodes are taken as shown in Figure 1 in which a typical biquadratic elements and the parameter space are sketched.
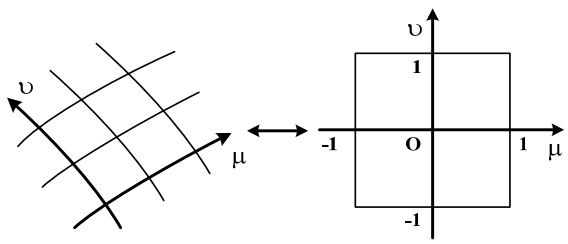

(a)

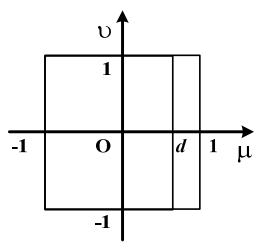

(b)

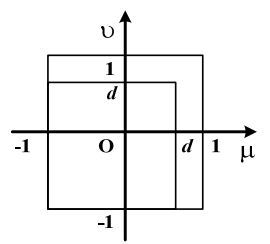

(c)

Figure 1: Bi-quadratic element and its parameter space: (a) continuous case, (b) single-edge case, (c) double-edge case

For appropriate treatment of the intersection line between two different boundaries, discontinuous elements are utilized around corners and edges as Brebbia [2] indicated, while conventional continuous elements are used elsewhere. The single-edge discontinuous elements are pertinent around edges or intersection lines and the double-edge discontinuous elements are suitable around corners. Throughout the computations, freedom nodes are taken at $d=2 / 3$ for both kinds of discontinuous elements.

Using the isoparametric bi-quadratic elements, geometry and variables on each element can be expressed in terms of basis functions

$$
[\overrightarrow{\boldsymbol{x}}, \varphi, q]=\sum_{\mathrm{m}=0}^{8} N_{\mathrm{m}}(\mu, v)\left[\overrightarrow{\boldsymbol{x}}_{m}^{j}, \varphi_{m}^{j}, q_{m}^{j}\right] \text { on } E_{\mathrm{j}} \text { with, } q=\varphi_{n}
$$

where $\vec{x}$ is the position vector of the boundary surface, the subscripts in variables designate the local node numbers, the ordering of which is depicted in Figure 2. Superscripts denote the number of the element on which variables are defined. Function $N_{m}$ is the product Lagrangian basis function, and $E_{\mathrm{j}}$ is the $\mathrm{j}^{\text {th }}$ element. By using this expression, the boundary integral equations are discretized.

To obtain the algebraic equations for the velocity potentials and their normal derivatives at each nodal point, the collocation method is applied. In this paper, collocation points correspond to nodal points. The discretized and collocated boundary integral equations form the following linear system of equations.

$$
\alpha\left(\vec{x}_{i}\right) \varphi\left(\vec{x}_{i}\right)=\sum_{j=1}^{N_{e}} \sum_{\mathrm{k}=0}^{8}\left[\varphi_{\mathrm{k}}^{\mathrm{j}} D_{\mathrm{k}}^{\mathrm{j}}\left(\vec{x}_{i}\right)-q_{\mathrm{k}}^{\mathrm{j}} S_{\mathrm{k}}^{\mathrm{j}}\left(\vec{x}_{i}\right)\right],
$$


where $D_{\mathrm{k}}^{\mathrm{j}}\left(\vec{x}_{i}\right)$ and $S_{\mathrm{k}}^{\mathrm{j}}\left(\vec{x}_{i}\right)$ are the influence coefficients due to the doublet and source distribution.

The numerical integration for influence coefficients due to source and doublet distribution is as follows: (1) regular integrals are calculated by a bi-directional Gauss-Legendre quadrature method; (2) singular integrals, in which the distance vanishes at the collocation point are completely desingularized by means of the rectangular polar coordinates mapping on each subdivision of parameter space and then Gauss-Legendre numerical integration is applied.

\subsection{Numerical radiation condition}

It is well known that the treatment of the radiation condition affects the entire solution and that it is closely related with the method of generating nonlinear waves in a numerical wave tank. Several kinds of numerical radiation condition are proposed for three-dimensional nonlinear diffraction problem. They may be categorized into two groups: wave damping zone technique (Boo and Kim [1], Kim et al. [11], Ferrant [4]) and decomposition method (Ferrant [5]).

In this paper, the following new radiation condition is proposed by combining these two methods as follows.

$$
\begin{aligned}
\frac{d \varphi(\overrightarrow{\mathrm{x}}, \mathrm{t})}{d \mathrm{t}}= & {[1-\gamma(\mathrm{x}, \mathrm{y})]\left\{-\frac{1}{2}|\nabla \varphi|^{2}-\mathrm{g} z+\left[\frac{\partial \varphi}{\partial z}-\nabla_{H} \varphi \cdot \nabla_{H} \zeta\right] \frac{\partial \varphi}{\partial z}\right\} } \\
& +\gamma(\mathrm{x}, \mathrm{y})\left\{\frac{d \varphi_{R F}(\overrightarrow{\mathrm{x}}, \mathrm{t})}{d t}-\beta\left[\varphi(\overrightarrow{\mathrm{x}}, \mathrm{t})-\varphi_{R F}(\overrightarrow{\mathrm{x}}, \mathrm{t})\right]\right\}, \quad \overrightarrow{\mathrm{x}} \in \mathrm{S}_{\mathrm{f}}, \\
\frac{d \zeta(\mathrm{x}, \mathrm{y}, \mathrm{t})}{d \mathrm{t}}= & {[1-\gamma(\mathrm{x}, \mathrm{y})]\left\{\frac{\partial \varphi}{\partial \mathrm{z}}-\nabla_{H} \varphi \cdot \nabla_{H} \zeta\right\} } \\
& +\gamma(\mathrm{x}, \mathrm{y})\left\{\frac{d \zeta_{R F}(\mathrm{x}, \mathrm{y}, \mathrm{t})}{d \mathrm{t}}-\beta\left[\zeta(\mathrm{x}, \mathrm{y}, \mathrm{t})-\zeta_{R F}(\mathrm{x}, \mathrm{y}, \mathrm{t})\right]\right\}, x \in \mathrm{S}_{\mathrm{f}}
\end{aligned}
$$

where $\gamma(\mathrm{x}, \mathrm{y})$ is named the Homotopy parameter and $\beta$ is a time constant to retrieve the incident waves. These equations must be understood as timedependent boundary conditions for a given grid point $x=(x, y, z)$. The subscript RF denotes a quantity of the Rienecker and Fenton wave (Rienecker and Fenton [16]).

The RF waves are given by

$$
\begin{gathered}
\varphi_{R F}(\mathrm{x}, \mathrm{z}, \mathrm{t})=\left(\mathrm{B}_{0}+c\right) \mathrm{x}+\sum_{j=1}^{\mathrm{N}} \mathrm{B}_{j} \frac{\cosh [j \mathrm{k}(\mathrm{z}+\mathrm{h})]}{\cosh (j \mathrm{kh})} \sin [j \mathrm{k}(\mathrm{x}-\mathrm{ct})] \\
\zeta_{R F}(\mathrm{x}, \mathrm{t})=\frac{\mathrm{a}_{0}}{2}+\sum_{j=1}^{\mathrm{N}} \mathrm{a}_{j} \cos [j \mathrm{k}(\mathrm{x}-\mathrm{ct})]
\end{gathered}
$$

where $\mathrm{c}$ and $\mathrm{k}$ are the phase speed and the wave number, respectively. The coefficients $\mathrm{B}_{j}, \mathrm{a}_{j}(j=0,1, \cdots, \mathrm{N})$ are determined so that the nonlinear free surface conditions and compatibility conditions are satisfied usually when the wave height and the wave period are specified. The error can be controlled by the truncation order $\mathrm{N}$ only, which is 40 in the present computation. 
Specifically, $\gamma(\mathrm{x}, \mathrm{y})$ is determined by the horizontal distance $\mathrm{R}_{x y}$ from the center of the cylinder in this paper as follows.

$$
\gamma(\mathrm{x}, \mathrm{y})=\left\{\begin{array}{cc}
0 & \text { if } \mathrm{R}_{x y} \leq \mathrm{R}_{D} \\
\sin ^{2}\left[\left(\mathrm{R}_{x y}-\mathrm{R}_{D}\right) / 2\left(\mathrm{R}_{T}-\mathrm{R}_{D}\right)\right] & \text { if } \mathrm{R}_{D} \leq \mathrm{R}_{x y} \leq \mathrm{R}_{T} \\
1 & \text { if } \mathrm{R}_{x y} \geq \mathrm{R}_{T}
\end{array}\right.
$$

The three zones above are named as the $\mathrm{D}$ (Diffraction) zone, the $\mathrm{T}$ (Transient) zone, and the RF (Rienecker and Fenton wave) zone. At the RF zone, equations (9) and (10) assert that the solution converges to the RF wave after several wave periods. The value of the RF wave potential is specified on radiation boundaries at all times. Around the body, however, the potential and the wave elevation are gradually set to zero at $t=0$ in order to avoid impulsive responses. The present method is compared with the work of Ferrant [4]. This scheme is able to describe nonlinear diffraction waves near the body accurately, and the incident waves are well recovered away from the body.

\subsection{Evaluation of the wave forces and moments}

In order to evaluate the wave forces and moments, we calculate as follows.

$$
(\overrightarrow{\mathrm{F}}, \overrightarrow{\mathrm{M}})=\int_{\mathrm{S}_{b}} \mathrm{p}(\overrightarrow{\mathrm{x}}, \mathrm{t})(\overrightarrow{\mathrm{n}}, \overrightarrow{\mathrm{r}} \times \overrightarrow{\mathrm{n}}) d \mathrm{~S}=-\rho \int_{\mathrm{S}_{b}}\left[\varphi_{t}+\frac{1}{2}|\nabla \varphi|^{2}+\mathrm{gz}\right](\overrightarrow{\mathrm{n}}, \overrightarrow{\mathrm{r}} \times \overrightarrow{\mathrm{n}}) d \mathrm{~S}
$$

Using Leinitz' rule, we can transform the surface integral of the time derivative of the velocity potential into sum of the time derivative of the surface integral of the velocity potential and the line integral term that is defined along the intersection line of the free surface and the body boundary.

A more orthodox method of solution is the use of the concept of acceleration potential. The time derivative of the velocity potential, $\partial \Phi / \partial t$, is of great concern in time simulation of fully nonlinear free surface waves around the offshore structures, particularly for the wave forces prediction. It can be estimated by using the backward time difference formula, which however leads to inaccurate results and unstable solutions. According to our understanding, we can also utilize the concept of the acceleration potential, $\Psi(\vec{x}, t)=\partial \Phi / \partial t+\nabla \Phi \cdot \nabla \Phi / 2$ which postulates the continuity of the normal acceleration. This idea was originally suggested in series of Tanizawa's works [21]. In this paper the procedure given by Tanizawa is borrowed and some implemental issues are explained. Thus we start from the boundary condition for the acceleration potential as follows.

$$
\frac{\partial}{\partial n}\left(\frac{\partial \Phi}{\partial t}\right)=\vec{A} \cdot \vec{n}-[(\nabla \Phi \cdot \nabla) \nabla \Phi] \cdot \vec{n}
$$

where $\vec{A}$ means the acceleration vector on the body boundary, which is zero in this case. The expression for the second term is stated for two-dimensions in Tanizawa [21]. For the three dimensional case, the equation of the particle acceleration in Fochesato et al. [6] can be utilized for the present purpose. Interested readers can refer to the paper by [6]. 


\subsection{Miscellaneous aspects}

In this study, the linear system of algebraic BEM equations is solved using the iterative solver GMRES (Generalized Minimal RESidual) originally devised by Saad and Schultz [17] which is known as a $N \log N$ method, where $N$ is the degree of freedom. For the time integration of the time-dependent system, the fourth-order Runge-Kutta (RK4) method with minimum truncation error (Ralston and Rabinowitz [15]) is adopted by considering accuracy and stability.

\subsection{Perturbation-based solution method}

In order to investigate systematically the overall capability and performance of the fully nonlinear method, the present study utilizes the perturbation-based solution scheme. Traditionally in it, the whole solution is expressed as follows.

$$
\begin{aligned}
& \Phi=\varepsilon \Phi^{(1)}+\varepsilon^{2} \Phi^{(2)}+\text { H.O.T. } \\
& \zeta=\varepsilon \zeta^{(1)}+\varepsilon^{2} \zeta^{(2)}+\text { H.O.T. }
\end{aligned}
$$

where $\varepsilon=k A_{I}$ is known as smallness parameter, when $k$ is the wavenumber and $A_{I}$ the amplitude of incident waves. The superscript means the order of the solution. H.O.T. means higher-order terms. As well-known, the free surface boundary conditions for the first-order of solution can be written as follows.

$$
\begin{aligned}
& \frac{\partial \zeta^{(1)}}{\partial t}=\frac{\partial \Phi^{(1)}}{\partial z} \text { at } z=0 \\
& \frac{\partial \Phi^{(1)}}{\partial t}=-g \zeta^{(1)} \text { at } z=0
\end{aligned}
$$

The second-order ones are of the following form.

$$
\begin{gathered}
\frac{\partial \zeta^{(2)}}{\partial t}=\frac{\partial \Phi^{(2)}}{\partial z}+\zeta^{(1)} \frac{\partial^{2} \Phi^{(1)}}{\partial z^{2}}-\nabla_{H} \Phi^{(1)} \cdot \nabla_{H} \zeta^{(1)} \text { at } z=0 \\
\frac{\partial \Phi^{(2)}}{\partial t}=-g \zeta^{(2)}-\zeta^{(1)} \frac{\partial^{2} \Phi^{(1)}}{\partial z \partial t}-\frac{1}{2}\left|\nabla \Phi^{(1)}\right|^{2} \text { at } z=0
\end{gathered}
$$

Here the atmospheric pressure has been set to zero for brevity.

The incident wave potentials for each order are known as:

$$
\begin{gathered}
\Phi_{I}^{(1)}=\frac{g}{k \omega} \frac{\cosh k(z+h)}{\cosh k h} \sin (k x-\omega t) \\
\Phi_{I}^{(2)}=\frac{3 g}{8 k^{2}} \frac{\cosh 2 k(z+h)}{\sinh ^{4} k h} \sin (2 k x-2 \omega t)
\end{gathered}
$$

The solution can be obtained by decomposition of the solution into the sum of incident and scattered parts. In the present study, the first- and second-order solutions can be compared with the fully nonlinear results.

\section{Numerical results}

While the present study is under development, this paper contains the first-order computation only. The second-order and fully nonlinear simulations are postponed to near future. Figure 2 shows the grid systems for the first-order and 
second-order solutions. An appropriate grid should resolve the evolution of incident and scattered wave for each order of solution, and so each grid has its own dimension.

An example of free surface shape is shown in Figure 3. The scattered part and total waves surface are separately displayed.
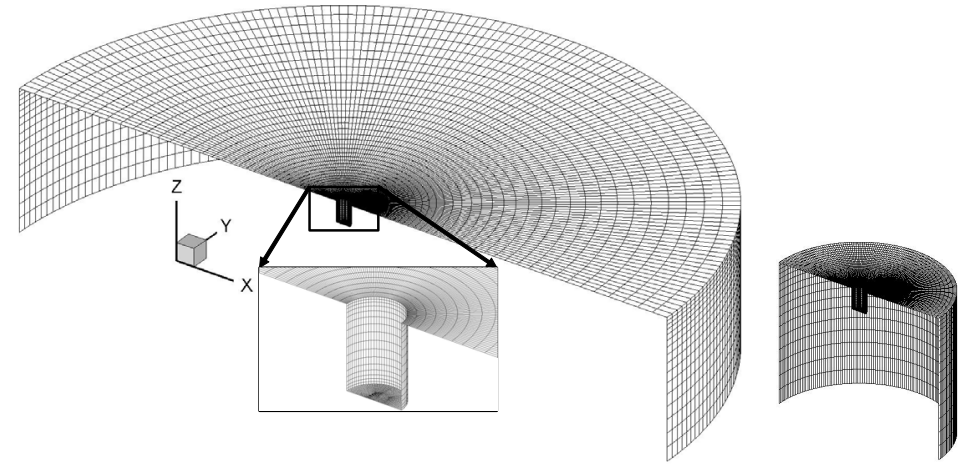

Figure 2: Grid systems for the first-order (left) and second-order (right) solutions.
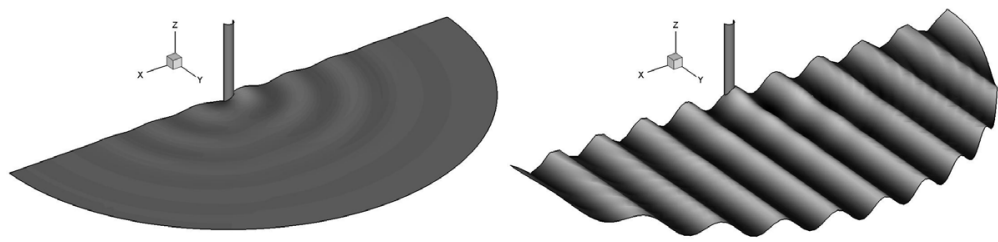

Figure 3: $\quad$ Free surface shape at $t=14 T$ of the first-order solution for the case of $k R=0.657$ (left-scattered part, right-total=incident + scattered).

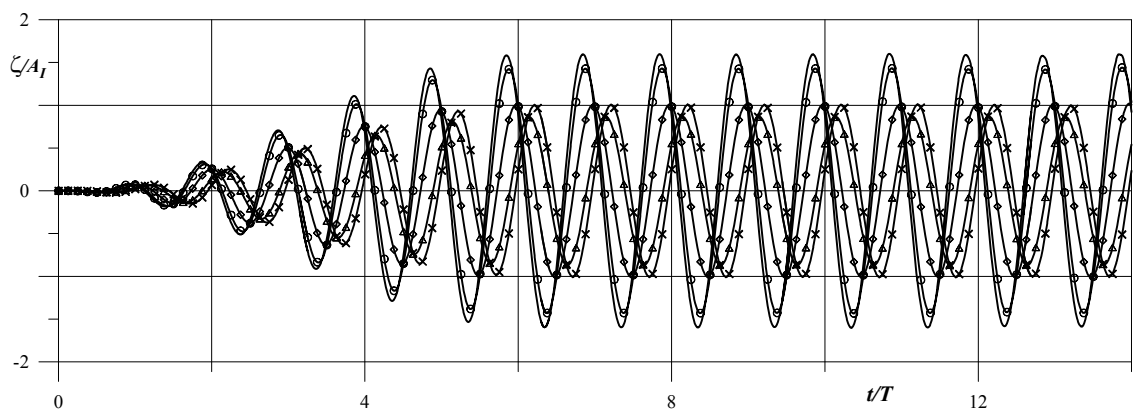

Figure 4: Time series of wave runups for the case of $k R=0.657$ (line: $\theta=0$, line with circle: $\theta=\pi / 4$, line with diamond: $\theta=\pi / 2$, line with triangle: $\theta=3 \pi / 4$, line with cross: $\theta=\pi$; the angle is so defined that $\theta=\pi$ corresponds to the lee side of the cylinder). 


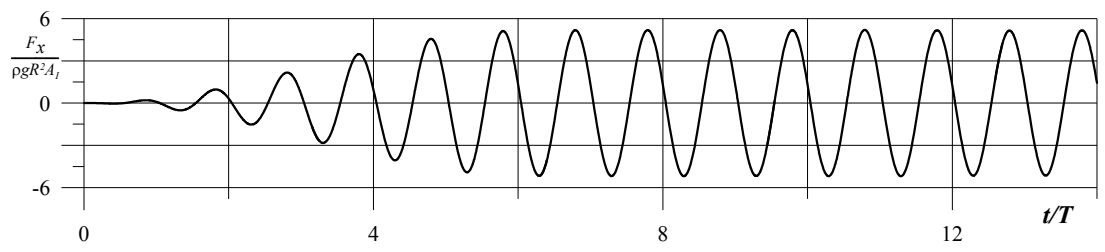

Figure 5: $\quad$ Time series of horizontal wave force for the case of $k R=0.657$.

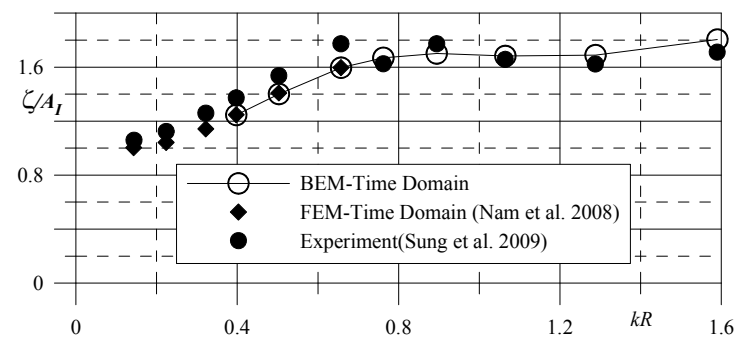

Figure 6: Comparison of wave runup at upstream point of the cylinder ( $r=R, \theta=0$ ) with numerical computation by FEM (Nam et al. [14]) and experiment (for the wave steepness, $H / \lambda=1 / 30$, Sung et al. [20]).

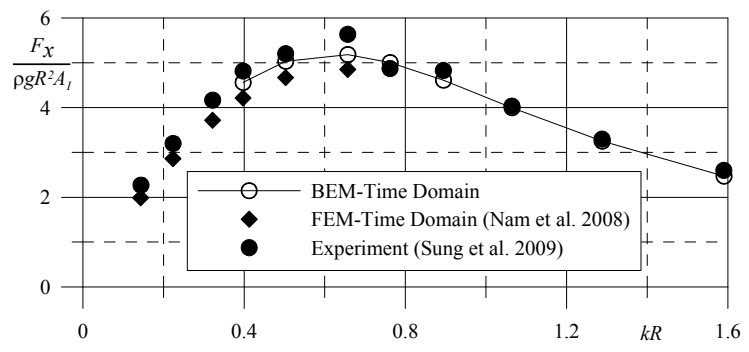

Figure 7: Comparison of horizontal wave force with numerical computation by FEM (Nam et al. [14]) and experiment (Sung et al. [20]).

Time series of wave elevations along the cylinder surface for the case of $k R=0.657$ are shown in Figure 4. Corresponding time series of horizontal wave force behaves as in Figure 5.

For qualitative comparison, a series of experiments has been carried out, and a detailed description of the experimental study can be referred to Sung et al. [20]. Comparison of present first-order computation with experiments is shown in Figure 6 and Figure 7. The experimental results were obtained for the case of intermediate wave steepness, $H / \lambda=1 / 30$, which can be regarded as 'linear case'. It can be concluded that the present method is fairly accurate for wave forces and run-ups on the whole when compared with experimental results and also with other numerical results. 
The second-order and fully nonlinear computation is postponed, and lastly the need to incorporate nonlinearity into the numerical solution is demonstrated. Figure 8 shows nonlinearity of the wave runup, wave forces, and moment, where time series behaves very differently from a pure sinusoidal pattern. Higher-order components seem to be strongly inherent in this case of $k R=0.657$ and $H / \lambda=1 / 10$, which will be analyzed more thoroughly with fully nonlinear computation.

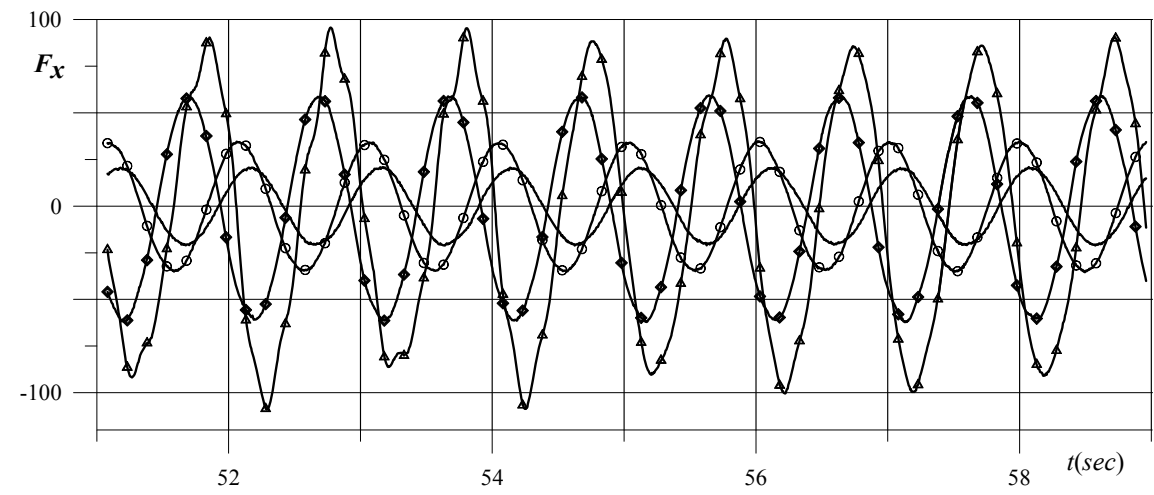

Figure 8: Experimental results of nonlinearity of the wave runup, wave forces and moment for the case of $k R=0.762$ (line: $H / \lambda=1 / 50$, line with circle: $H / \lambda=1 / 30$, line with diamond: $H / \lambda=1 / 16$, line with triangle: $H / \lambda=1 / 10)$.

\section{Summary and concluding remarks}

This paper presented recent accomplishments of the development of the Boundary Element Method (BEM) for nonlinear waves around offshore structures and resulting wave forces. Basic characteristics of the present method of solution were briefly stated with a state-of-the-art review on computational methods of prediction for nonlinear wave forces on offshore structures by using BEM. The perturbation-based first- and second-order solution methods were stated for comparison with the fully nonlinear scheme. In order to investigate the capability of the present method, diffraction problem of a truncated circular cylinder is simulated. The present paper includes the numerical results with firstorder solution only, while second-order and fully nonlinear methods are being developed and complete solutions are expected to come up soon. It is found out that the present method is fairly accurate for wave forces and run-ups on the whole when compared with experimental results and also with other numerical results. It is also demonstrated that highly nonlinear features manifest clearly for incident waves with high steepness, i.e. $H / \lambda=1 / 16$ or $H / \lambda=1 / 10$. These phenomena will be dealt with more thoroughly by fully nonlinear simulation in near future. 


\section{Acknowledgements}

The author acknowledges support from a grant from the Ministry of Knowledge and Economics of Korea through the principal R\&D program: "Core Methodologies for Design of LNG FSRU's Structure and Mooring System.”

\section{References}

[1] Boo, S.Y. and Kim, C.H. "Fully nonlinear diffraction due to a vertical circular cylinder in a 3-D HOBEM numerical wave tank", Proceedings of the Sixth International Offshore and Polar Engineering Conference, 1996, 3, 23-30.

[2] Brebbia, C.A. Topics in boundary element research. Vol. 1: Basic principles and applications, 1984 (Springer-Verlag, Berlin).

[3] Dommermuth, D.G., Yue, D.K.P., Lin, W.M., Rapp, R.J., Chan, E.S. and Melville, W.K. "Deep-water plunging breakers: A comparison between potential theory and experiments", Journal of Fluid Mechanics, 1988, 184, 267-288.

[4] Ferrant, P. "Time domain computation of nonlinear diffraction loads upon three dimensional floating bodies", Proceedings of the Fifth International Offshore and Polar Engineering Conference, 1995, 3, 280-288.

[5] Ferrant, P. "Runup on a cylinder due to waves and current: Potential flow solution with fully nonlinear boundary conditions", Proc., 8th Int. Offshore and Polar Engineering Conf., Vol. 3, 1998, 332 339.

[6] Fochesato, C, Grilli, ST, and Guyenne, P. "Note on nonorthogonality of local curvilinear coordinates in a 3-D Boundary Element Method," Intl. J. Num. Methods Fluids, Vol. 48, 2005, 305-324.

[7] Grilli, S.T., Dias, F., Guyenne, P., Fochesato, C. and F. Enet (2008), "Progress In Fully Nonlinear Potential Flow Modeling of 3D Extreme Ocean Waves." Chapter in Advances in Numerical Simulation of Nonlinear Water Waves (Series in Advances in Coastal and Oc. Engng.). World Scientific Pub., 2009, 55 pps. (n Press)

[8] Grilli, S.T., P. Guyenne and F. Dias (2001), "A fully non-linear model for three-dimensional overturning waves over an arbitrary bottom," Intl. J. Num. Methods Fluids, 35, pp. 829-867.

[9] Guyenne, P. and Grilli, S.T. (2006), "Numerical study of three-dimensional overturning waves in shallow water," J. Fluid Mech., 547, pp. 361-388.

[10] Kim, C.H. "Recent progress in numerical wave tank research", Proceedings of the Fourth International Offshore and Polar Engineering Conference, 1995, 3, 1-9.

[11] Kim, M.H., Celebi, M.S. and Park, J.C. "Nonlinear wave-body interactions by numerical wave tank simulations", Proceedings of the third international conference on hydrodynamics, 1998, 1, 139-144.

[12] Kristiansen, T., Baarholm, R. and Stansberg, C. T. "Validation of Secondorder Analysis in Predicting Diffracted Wave Elevation around a Vertical Circular Cylinder", Proc., 14th ISOPE Conference, 2004, 342 349. 
[13] Longuet-Higgins, M.S. and Cokelet, E.D. "The deformation of steep surface waves on water, I. A numerical method of computations", Proc. Roy. Soc. London, 1976, Ser. A, 350, 1-26.

[14] Nam, B.W., Sung, H.G., Kim, Y.S., and Hong, S.Y. "Linear and high-order computations for wave field around truncated cylinder in waves using finite element method", Journal of Ships \& Ocean Engineering, Vol 46, pp. 4352.

[15] Ralston, A. and Rabinowitz, P. A first course in numerical analysis, $2^{\text {nd }}$ ed., 1978 (McGraw-Hill Book Company).

[16] Rienecker, M.M. and Fenton J.D. "A Fourier approximation method for steady water waves", Journal of Fluid Mechanics, 1981, Vol. 104, pp. 119 137

[17] Saad, Y. and Schultz, M.H. "GMRES: A generalized minimal residual algorithm for solving nonsymmetric linear systems", SIAM J. Sci. Stat. Comput., 1986, 7, 856-869.

[18] Sung, H.G. A numerical analysis of nonlinear diffraction problem in three dimensions by using higher-order boundary element method, 1999, Ph.D. Thesis (Seoul National University).

[19] Sung, H.G., Hong, S.Y. and Choi, H.S. "Evaluation of non-linear wave forces on a fixed body by the higher-order boundary element method", Journal of Mechanical Engineering Science (Proceedings of the Institution of Mechanical Engineers Part C), 2000, 214, 825-839.

[20] Sung, H.G., Nam, B.W., Hong, S.Y., Kim, Y.S., Kim, J.H. Kim, and Choi, S.K. "Experimental study of wave forces on a truncated circular cylinder in extreme seas", 2009, In preparation.

[21] Tanizawa K. Nonlinear simulation of floating bodies in waves, Proceedings of the Sixth International Offshore and Polar Engineering Conference 1996; 3: 414-420.

[22] Xü, H. Numerical study of fully nonlinear water waves in three dimensions, 1992, Ph.D. Dissertation (MIT). 